\title{
Toward Understanding Heterogeneity in Computing ${ }^{\star}$
}

\author{
Arnold L. Rosenberg and Ron C. Chiang \\ Electrical \& Computer Engineering, Colorado State Univ., Fort Collins, CO 80523, USA \\ \{rsnbrg,ron.chiang\}@colostate.edu
}

\begin{abstract}
Heterogeneity complicates the efficient use of multicomputer platforms, but does it enhance their performance? their cost effectiveness? How can one measure the power of a heterogeneous assemblage of computers ("cluster," for short), both in absolute terms (how powerful is this cluster) and relative terms (which cluster is the most powerful)? What makes one cluster more powerful than another? Is one better off with a cluster that has one super-fast computer and the rest of just "average" speed or with a cluster all of whose computers are "moderately" fast? If you could replace just one computer in your cluster with a faster one, which computer would you choose: the fastest? the slowest? How does one even ask questions such as these in a formal, yet tractable manner? A framework is proposed, and some answers are derived, a few rather surprising. Three highlights: (1) If one can replace only one computer in a cluster by a faster one, it is provably (almost) always most advantageous to replace the fastest one. (2) If the computers in two clusters have the same mean speed, then, empirically, the cluster with the larger variance in speed is (almost) always the faster one. (3) Heterogeneity can actually lend power to a cluster!
\end{abstract}

\section{Motivation and Background}

Modern multicomputer platforms are heterogeneous: their constituent computers vary in computational powers, and they often intercommunicate over layered networks of varying speeds [12]. One observes substantial heterogeneity in modern platforms such as: clusters [2, 21]; modalities of Internet-based computing [20] such as grid computing [9, 14], global computing [11], volunteer computing [16], and cloud computing [10]. The difficulty of scheduling complex computations on heterogeneous platforms greatly complicates the challenge of high performance computing in modern environments. In 1994, the first author noted the need for better understanding of the scheduling implications of heterogeneity via rigorous analyses [23]. There has since been an impressive amount of first-rate work on this topic-focusing largely on collective communication [3, 4, 8, 15, 17, 22, 24], but also studying important scheduling issues $[1,5,6,7,13,18]$. That said, sources such as [1] show that there is still much to learn about this important topic -including the questions in the abstract.

This research was supported in part by NSF Grants CNS-0615170 and CNS-0905399. 


\section{1 "Understanding" Heterogeneity}

We have access to $n+1$ computers: the server $C_{0}$ and a cluster $\mathcal{C}$ comprising $n$ computers, $C_{1}, \ldots, C_{n}$, which may differ dramatically in computing powers. (We call $\mathcal{C}$ a "cluster" for convenience: the $C_{i}$ may be geographically dispersed and more diverse in power than that term usually connotes.) We have a uniform workload, and each $C_{i}$ can complete one unit of work in $\rho_{i}$ time units. ${ }^{1}$ The vector $\left\langle\rho_{1}, \ldots, \rho_{n}\right\rangle$ is $\mathcal{C}$ 's (heterogeneity) profile. For convenience:

- we index the $C_{i}$ in nonincreasing order of power, so that $\rho_{1} \geq \cdots \geq \rho_{n}$;

- we normalize the $\rho_{i}$ so that the slowest computer, $C_{1}$, has $\rho$-value $\rho_{1}=1$. (This "power indexing" only identifies computers, so normalization cannot lead to problems.)

We study heterogeneity within the context of the questions in the abstract. How does one deal with such questions rigorously? When can one say that cluster $\mathcal{C}$ "outperforms" (or, is more "powerful" than) cluster $\mathcal{C}^{\prime}$ ? We invoke the framework of a remarkable result from [1] that characterizes all optimal solutions to a simple scheduling problem for heterogeneous clusters. We thereby isolate the heterogeneity of $\mathcal{C}$ and $\mathcal{C}^{\prime}$ as the only respect in which they differ: both are performing the same computation optimally, given their respective resources.

Highlight results: Among our several results, three stand out. (1) If one can replace only one computer in a cluster by a faster one, then it is (almost) always most advantageous to replace the fastest computer. This is always true for "additive" speedups (Theorem 3) and almost always for "multiplicative" ones (Theorem 4). (2) If the computers in two $n$ computer clusters have the same mean speed, then the cluster with the larger variance in computers' speeds is (almost) always the faster one (Section 3.2). This is always true for 2computer clusters; for other sizes, the advantage takes hold when the difference in variances is sufficiently large. (3) Heterogeneity can actually lend power to a cluster! (Corollary 1).

\subsection{The Cluster-Exploitation Problem}

$C_{0}$ has $W$ units of work consisting of mutually independent tasks of equal sizes and complexities. $^{2}$ (Such workloads arise in diverse applications, e.g., data smoothing, pattern matching, ray tracing, Monte-Carlo simulations, chromosome mapping [16, 19, 25].) The tasks' (common) complexity can be an arbitrary function of their (common) size. $C_{0}$ must distribute a "package" of work to each $C_{i} \in \mathcal{C}$, in a single message. Each unit of work produces $\delta \leq 1$ units of results; each $C_{i}$ must return the results from its work, in one message, to $C_{0}$. At most one intercomputer message can be in transit at a time. Consider the following problem.

The Cluster-Exploitation Problem (CEP). $C_{0}$ must complete as many units of work as possible on cluster $\mathcal{C}$ within a given lifespan of $L$ time units.

\footnotetext{
${ }^{1}$ Note that faster computers have smaller $\rho$-values.

2 "Size" quantifies specification; "complexity" quantifies computation.
} 
A unit of work is "complete" once $C_{0}$ has transmitted it to a $C_{i}$, and $C_{i}$ has computed the unit and transmitted its results to $C_{0}$. We call a schedule for the CEP a worksharing protocol.

The main focus of our study is on experimental illustration and elucidation of the analytical results we derive; therefore, we relegate all proofs of new results to an appendix.

\section{Worksharing Protocols and Work Production}

\subsection{The Architectural Model [12]}

We assume that $\mathcal{C}$ 's computers are (architecturally) balanced: if $\rho_{i}<\rho_{j}$, then every one of $C_{i}$ 's subsystems (memory, I/O, etc.) is faster, by the factor $\rho_{j} / \rho_{i}$, than the corresponding subsystem of $C_{j}$. Computers intercommunicate over networks with a uniform transit rate of $\tau$ time units to send one unit of work from any $C_{i}$ to any $C_{j}$. Before injecting a message $\mathcal{M}$ into the network, $C_{i}$ packages $\mathcal{M}$ (e.g., packetizes, compresses, encodes) at a rate of $\pi_{i}$ time units per work unit. When $C_{j}$ receives $\mathcal{M}$, it unpackages it, also at a rate of $\pi_{j}$ time units per work unit. ${ }^{3}$ We ignore the fixed costs associated with transmitting $\mathcal{M}$ - the end-to-end latency of the first packet and the set-up cost - because their impacts fade over long lifespans L. A final important feature: At most one intercomputer message can be in transit at any moment. The following table provides intuition about the sizes of the model's parameters.

\begin{tabular}{|ll|rl|}
\hline \multicolumn{1}{c}{ Parameter } & \multicolumn{3}{c|}{ Wall-Clock Time/Rate } \\
\hline \hline Transit rate (pipelined network): & $\tau$ & $1 \mu \mathrm{sec}$ & per work unit \\
\hline Packaging rate: & $\pi$ & $10 \mu \mathrm{sec}$ & per work unit \\
\hline Result-size rate: & $\delta$ & 1 work unit & per work unit \\
\hline
\end{tabular}

We thus envisage an environment (workload plus platform) in which several linear relationships hold. The cost of transmitting work grows linearly with the total amount of work performed: formally, there are constants $\kappa, \kappa^{\prime}$ such that transmitting $w$ units of work takes $\kappa w$ time units, and receiving the results from that work takes $\kappa^{\prime} w$ time units. These relationships allow us to measure both time and message-length in the same units as work.

Note. A linear relationship between task-size and task-complexity does not limit tasks' (common) complexity as a function of their (common) size: $\kappa$ is just the ratio of the fixed task size to the complexity of a task of that size.

\subsection{Worksharing Protocols [1]}

One remote computer. $C_{0}$ shares $w$ units of work with a single $C_{i}$ via the process summarized in the following action/time diagram (not to scale):

\footnotetext{
${ }^{3}$ We equate packaging and unpackaging times; this is consistent with most actual architectures.
} 


\begin{tabular}{|c||c||c|c|c||c||c|}
\hline $\begin{array}{c}C_{0} \text { packages } \\
\text { work for } C_{i}\end{array}$ & $\begin{array}{c}\text { work is } \\
\text { in transit }\end{array}$ & $\begin{array}{c}C_{i} \text { receives } \\
\text { the work }\end{array}$ & $\begin{array}{c}C_{i} \text { computes } \\
\text { the work }\end{array}$ & $\begin{array}{c}C_{i} \text { packages } \\
\text { its results }\end{array}$ & $\begin{array}{c}\text { results are } \\
\text { in transit }\end{array}$ & $\begin{array}{c}C_{0} \text { receives } \\
\text { the results }\end{array}$ \\
\hline$\pi_{0} w$ & $\tau w$ & $\pi_{i} w$ & $\rho_{i} w$ & $\pi_{i} \delta w$ & $\tau \delta w$ & $\pi_{0} \delta w$ \\
\hline
\end{tabular}

Multiple remote computers. A pair of ordinal-indexing schemes for $\mathcal{C}$ 's computers (to complement the power-indexing) helps us orchestrate communications while solving the CEP. The startup indexing specifies the order in which $C_{0}$ transmits work within $\mathcal{C}$; it labels the computers $C_{s_{1}}, \ldots, C_{s_{n}}$, to indicate that $C_{s_{i}}$ receives work-hence, begins working-before $C_{s_{i+1}}$. Dually, the finishing indexing labels the computers $C_{f_{1}}, \ldots, C_{f_{n}}$, to specify the order in which they return their results to $C_{0}$. Protocols proceed as follows.

1. Transmit work. $C_{0}$ prepares and transmits $w_{s_{1}}$ units of work for $C_{s_{1}}$. It immediately prepares and sends $w_{s_{2}}$ units of work to $C_{s_{2}}$ via the same process. Continuing thus, $C_{0}$ supplies each $C_{s_{i}}$ with $w_{s_{i}}$ units of work seriatim-with no intervening gaps.

2. Compute. As soon as $C_{i}$ receives its work from $C_{0}$, it unpackages and performs the work.

3. Transmit results. As soon as $C_{i}$ completes its work, it packages its results and transmits them to $C_{0}$.

We choose work-allocations $w_{i}$ so that, with no gaps, $\mathcal{C}$ 's computers:

- receive work and compute in the startup order $\Sigma=\left\langle s_{1}, \ldots, s_{n}\right\rangle$;

- complete work and transmit results in the finishing order $\Phi=\left\langle f_{1}, \ldots, f_{n}\right\rangle$;

- complete all work and communications by time $L$.

The described protocol is summarized in diagram (2.1) (not to scale). Note that in this diagram, $\Sigma$ and $\Phi$ coincide: $(\forall i)\left[f_{i}=s_{i}\right]$. This is not true in general - cf. [1]-but protocols that share this coincidence are quite special within the context of the CEP.

\begin{tabular}{|c|c|c|c|c|c|c|}
\hline$C_{0}$ & $\begin{array}{c}\text { sends } \\
\text { work to } C_{1}\end{array}$ & $\begin{array}{c}\text { sends } \\
\text { work to } C_{2}\end{array}$ & $\begin{array}{c}\text { sends } \\
\text { work to } C_{3}\end{array}$ & & & \\
\hline & $\left(\pi_{0}+\tau\right) w_{1}$ & $\left(\pi_{0}+\tau\right) w_{2}$ & $\left(\pi_{0}+\tau\right) w_{3}$ & & & \\
\hline$C_{1}$ & waits & \multicolumn{2}{|c|}{ processes } & results & & \\
\hline & & \multicolumn{2}{|c|}{$\left(\pi_{1}+\rho_{1}\right) w_{1}$} & $\left(\pi_{1}+\tau\right) \delta w_{1}$ & & \\
\hline$C_{2}$ & waits & waits & \multicolumn{2}{|c|}{ processes } & results & \\
\hline & & & \multicolumn{2}{|c|}{$\left(\pi_{2}+\rho_{2}\right) w_{2}$} & $\left(\pi_{2}+\tau\right) \delta w_{2}$ & \\
\hline$C_{3}$ & waits & waits & waits & \multicolumn{2}{|c|}{ processes } & results \\
\hline & & & & \multicolumn{2}{|c|}{$\left(\pi_{3}+\rho_{3}\right) w_{3}$} & $\left(\pi_{3}+\tau\right) \delta w_{3}$ \\
\hline
\end{tabular}

\subsection{Protocols that Solve the CEP Optimally}

The FIFO protocol is defined by coincident startup and finishing indexings $(\Sigma=\Phi)$, as in (2.1). Provided only that $L$ is large enough, FIFO protocols solve the CEP optimally [1]. 
Theorem 1 ([1]). Over any sufficiently long lifespan L, for any heterogeneous cluster $\mathcal{C}$ - no matter what its heterogeneity profile:

1. FIFO worksharing protocols provide optimal solutions to the CEP.

2. $\mathcal{C}$ is equally productive under every FIFO protocol, i.e., under all startup indexings.

Because FIFO protocols solve the CEP optimally for every heterogeneity profile, we use these solutions as our vehicle for studying clusters' heterogeneity.

\subsection{Two Ways to Measure a Cluster's Computing Power}

2.4.1 The $X$-measure and work production. The obvious way of using the CEP to measure a cluster $\mathcal{C}$ 's computing power is to determine how much work $\mathcal{C}$ completes in $L$ time units. The coda of Theorem 1 in [1] does this via an explicit expression. To simplify expressions, let $A=\pi+\tau$ and $B=1+(1+\delta) \pi$; see Table 1 .

\begin{tabular}{|c|c|c|}
\hline \multicolumn{3}{|c|}{ Sample Values for Perspective } \\
\hline Quantity & Wall-Clock Time $/ \mathrm{H}$ & \\
\hline$A=\pi+\tau:$ & $11 \mu \mathrm{sec}$ & per work unit \\
\hline$B=1+(1+\delta) \pi$ & (per-task time) $+11 \times 10^{-6} \mathrm{sec}$ & per work unit \\
\hline$B$ with coarse $(1 \mathrm{sec} /$ task $)$ tasks & $1.000011 \mathrm{sec}$ & per work unit \\
\hline$B$ with finer $(0.1 \mathrm{sec} /$ task $)$ tasks & $0.100011 \mathrm{sec}$ & per work unit \\
\hline
\end{tabular}

Table 1: Sample parameter values.

Theorem 2 ([1]). Let $\mathcal{C}$ have profile $\mathrm{P}=\left\langle\rho_{1}, \ldots, \rho_{n}\right\rangle$. Letting

$$
X(\mathrm{P})=\sum_{i=1}^{n} \frac{1}{B \rho_{i}+A} \cdot \prod_{j=1}^{i-1} \frac{B \rho_{j}+\tau \delta}{B \rho_{j}+A}
$$

the asymptotic work completed by $\mathcal{C}$ under the FIFO protocol is $W(L ; \mathrm{P})=\frac{1}{\tau \delta+1 / X(\mathrm{P})} \cdot L$.

Because $X(\mathrm{P})$ "tracks" $W(L ; \mathrm{P})$, in that $X\left(\mathrm{P}_{1}\right) \geq X\left(\mathrm{P}_{2}\right)$ if and only if $W\left(L ; \mathrm{P}_{1}\right) \geq W\left(L ; \mathrm{P}_{2}\right)$, we use $X(\mathrm{P})$ as our primary measure of $\mathcal{C}$ 's computing power.

In Section A, we verify that FIFO protocols allocate work to $\mathcal{C}$ 's computers in proportion to their speeds. This is a sort of "reality check" on our model, because intuition strongly suggests that optimal work allocations must be proportional. 
2.4.2 The Homogeneous-Equivalent Computing Rate (HECR). $X(\mathrm{P})$ is a viable and tractable measure but not very perspicuous. We propose, therefore, the following alternative measure for a heterogeneous cluster $\mathcal{C}$ with profile $\mathrm{P}=\left\langle\rho_{1}, \ldots, \rho_{n}\right\rangle$. Consider a homogeneous cluster $\mathcal{C}^{(\rho)}$, with profile $\mathrm{P}^{(\rho)}=\langle\rho, \ldots, \rho\rangle$ for some $\rho \leq 1$. $\mathcal{C}$ 's homogeneous-equivalent computation rate $(\mathrm{HECR}), \rho_{\mathcal{C}}$, is the largest $\rho$ such that $X\left(\mathrm{P}^{\left({ }^{(}\right)}\right) \geq X(\mathrm{P}){ }^{4}$

Proposition 1. ${ }^{5} \quad \rho_{\mathcal{C}}=\frac{A-\tau \delta}{B-(1-(A-\tau \delta) X(\mathrm{P}))^{1 / n} B}-\frac{A}{B}$.

The HECR measure "in action." We illustrate HECRs as performance measures by focusing on two $n$-computer heterogeneous clusters, which are identified via their profiles.

For integer function $f$, abbreviate the sequence $\langle f(1), \ldots, f(n)\rangle$ via the notation $\left\langle\left. f(i)\right|_{i=1} ^{n}\right\rangle$.

Cluster $\mathcal{C}_{1}$ has profile $\mathrm{P}_{1}^{(n)}=\left\langle\left.(1-(i-1) / n)\right|_{i=1} ^{n}\right\rangle$, meaning that each $\rho_{i}=1-(i-1) / n$; cluster $\mathcal{C}_{2}$ has profile $\mathrm{P}_{2}^{(n)}=\left\langle\left.(1 / i)\right|_{i=1} ^{n}\right\rangle$, meaning that each $\rho_{i}=1 / i$. Note that the speeds of $\mathcal{C}_{1}$ 's computers are spread evenly in the range $[1 / n, 1]$, while the speeds of $\mathcal{C}_{2}$ 's computers are weighted in the faster half of this range, namely, $[1 / n, 1 / 2]$. When $n=8$, for example, $\mathrm{P}_{1}^{(8)}=\left\langle 1, \frac{7}{8}, \ldots, \frac{1}{8}\right\rangle$, and $\mathrm{P}_{2}^{(8)}=\left\langle 1, \frac{1}{2}, \ldots, \frac{1}{8}\right\rangle$. Note that most of $\mathcal{C}_{2}$ 's computers are faster than their counterparts in $\mathcal{C}_{1}$, a fact that should be reflected in the HECR-values of the two clusters: $\mathcal{C}_{1}$, being slower than $\mathcal{C}_{2}$, should have a larger HECR-value (Proposition 1). Table 2 presents the HECR-values of three instantiations of clusters $\mathcal{C}_{1}$ and $\mathcal{C}_{2}$ : with 8 , 16, and 32 computers. As expected, $\mathcal{C}_{1}$ 's HECR-value is larger than $\mathcal{C}_{2}$ 's for each cluster size. Additionally, because all but one of $\mathcal{C}_{2}$ 's computers have $\rho$-values $\leq 1 / 2$, while half of $\mathcal{C}_{1}$ 's computers have $\rho$-values $>1 / 2$, we know intuitively that $\mathcal{C}_{2}$ 's speed advantage over $\mathcal{C}_{1}$ should increase with larger instantiations of the two clusters. Indeed, the entries in Table 2 demonstrate this trend, as the ratio of $\mathcal{C}_{2}$ 's HECR-value to $\mathcal{C}_{1}$ 's improves from roughly 1.7 for 8 computers to roughly 2.6 for 16 computers to more than 4 for 32 computers.

\begin{tabular}{|c|c||c|c|c|}
\hline \multirow{2}{*}{ Cluster } & \multicolumn{2}{|c||}{ Profile } & \multicolumn{3}{c|}{ Number of Computers } \\
\cline { 3 - 5 } & & 8 & 16 & 32 \\
\hline \hline $\mathcal{C}_{1}$ & $\left\langle\left.(1-(i-1) / n)\right|_{i=1} ^{n}\right\rangle$ & 0.366 & 0.298 & 0.251 \\
\hline $\mathcal{C}_{2}$ & $\left\langle\left.(1 / i)\right|_{i=1} ^{n}\right\rangle$ & 0.216 & 0.116 & 0.060 \\
\hline
\end{tabular}

Table 2: HECR values for sample competing heterogeneous clusters

\footnotetext{
${ }^{4}$ Because we use the value of $\rho$ to calibrate a heterogeneous cluster's power, we must violate our normalization convention and allow $\rho$ to assume any value $\leq 1$.

${ }^{5}$ Proof appears in Section B.1.
} 


\section{What Determines a Cluster's Power?}

\subsection{Speeding up a Cluster Optimally}

We study how to speed up a cluster "optimally." After showing that replacing any of $\mathcal{C}$ 's computers by a faster one always enhances $\mathcal{C}$ 's power, we consider which $C_{i} \in \mathcal{C}$ is the most advantageous one to replace. We study both additive speed-ups, wherein a computer with speed $\rho$ is replaced by one with speed $\rho-\varphi$, and multiplicative speed-ups, wherein a computer with speed $\rho$ is replaced by one with speed $\psi \rho$; of course, $0<\varphi<\rho_{n}$ and $0<\psi<1$.

3.1.1 Faster clusters complete more work. Speedups always matter for FIFO protocols.

Proposition 2. ${ }^{6}$ FIFO protocols complete more work on faster clusters; i.e., given profiles $\mathrm{P}=\left\langle\rho_{1}, \ldots, \rho_{i-1}, \rho_{i}, \rho_{i+1}, \ldots, \rho_{n}\right\rangle$ and $\mathrm{P}^{\prime}=\left\langle\rho_{1}, \ldots, \rho_{i-1}, \rho_{i}^{\prime}, \rho_{i+1}, \ldots, \rho_{n}\right\rangle:$ if $\rho_{i}^{\prime}<\rho_{i}$, then for all $L, W\left(L ; \mathrm{P}^{\prime}\right)>W(L ; \mathrm{P})$.

3.1.2 Which computer should one speed up? Say that one has resources to replace only one of cluster $\mathcal{C}$ 's computers by a faster one - or, equivalently, to speed up a single computer. Which computer should one choose? We focus on a cluster $\mathcal{C}$ whose heterogeneity profile is $\mathrm{P}=\left\langle\rho_{1}, \ldots, \rho_{n}\right\rangle$, where each $\rho_{k} \geq \rho_{k+1}$. Let $i$ and $j>i$ be two of $\mathcal{C}$ 's power indices. We compare the benefits of speeding up $C_{i}$ vs. speeding up $C_{j}$. Of course, in order for this question to make sense, we must have $\rho_{i}>\rho_{j}$, i.e., a strict inequality between $\rho_{i}$ and $\rho_{j}$. We answer this question twice - once for additive speedups and once for multiplicative ones.

The analyses that embody our comparisons are simplified if we require $\mathcal{C}$ to employ a startup ordering $\Sigma$ from a specific class - even though Theorem 1.2 assures us that $\Sigma$ has no impact on $W(L ; \mathrm{P})$. Specifically, we have $\mathcal{C}$ employ a startup ordering $\Sigma=\left\langle s_{1}, \ldots, s_{n-1}, s_{n}\right\rangle$ for which $s_{n}=i$ and $s_{n-1}=j$. Under such an ordering, we can rewrite expression (2.2) for $X(\mathrm{P})$ in the following convenient way, using two quantities that are independent of $\rho_{i}$ and $\rho_{j}$ and that, importantly, are both positive.

$$
X(\mathrm{P})=\frac{A+B\left(\rho_{s_{n-1}}+\rho_{s_{n}}\right)+\tau \delta}{A^{2}+A B\left(\rho_{s_{n-1}}+\rho_{s_{n}}\right)+B^{2} \rho_{s_{n-1}} \rho_{s_{n}}} \cdot Y(\mathrm{P})+Z(\mathrm{P})
$$

where

$$
Y(\mathrm{P})=\prod_{k=1}^{n-2} \frac{B \rho_{s_{k}}+\tau \delta}{B \rho_{s_{k}}+A} \quad \text { and } \quad Z(\mathrm{P})=X\left(\rho_{s_{1}}, \ldots, \rho_{s_{n-2}}\right)
$$

The fact that a faster cluster completes more work than a slower one suggests that we compare competing heterogeneity profiles, $\mathrm{P}$ and $\mathrm{P}^{\prime}$, via their work ratio, $W\left(L ; \mathrm{P}^{\prime}\right) / W(L ; \mathrm{P})$.

A. The additive-speedup scenario. We compare two profiles: $\mathrm{P}^{(i)}$ is obtained by speeding up the slower computer (of the two we are focusing on), $C_{i} ; \mathrm{P}^{(j)}$ is obtained by speeding up

\footnotetext{
${ }^{6}$ Proof appears in Section B.2.
} 
the faster computer, $C_{j}$. Both speedups are by the additive term $\varphi<\rho_{n}$. (This inequality ensures that we can speed up any of $\mathcal{C}$ 's computers by the term $\varphi$.)

$$
\begin{aligned}
& \mathrm{P}^{(i)}=\left\langle\rho_{1}, \ldots, \rho_{i-1}, \quad \rho_{i}-\varphi, \quad \rho_{i+1}, \ldots, \rho_{j-1}, \quad \rho_{j}, \quad \rho_{j+1}, \ldots, \rho_{n}\right\rangle \\
& \mathrm{P}^{(j)}=\left\langle\rho_{1}, \ldots, \rho_{i-1}, \quad \rho_{i}, \quad \rho_{i+1}, \ldots, \rho_{j-1}, \quad \rho_{j}-\varphi, \quad \rho_{j+1}, \ldots, \rho_{n}\right\rangle
\end{aligned}
$$

Theorem 3. ${ }^{7}$ Under the additive-speedup scenario, the most advantageous single computer to speed up is $\mathcal{C}$ 's fastest computer.

Additive speedup "in action." We compare $\mathrm{P}^{(i)}$ and $\mathrm{P}^{(j)}$ via the work ratios $W\left(L ; \mathrm{P}^{(i)}\right) / W(L ; \mathrm{P})$ and $W\left(L ; \mathrm{P}^{(j)}\right) / W(L ; \mathrm{P})$. Proposition 2 assures us that both ratios exceed 1 .

We illustrate Theorem 3 "in action" by considering the optimal sequence of additive speedups when we begin with the 4 -computer heterogeneous cluster $\mathcal{C}$ whose profile is $\mathrm{P}=\left\langle 1, \frac{1}{2}, \frac{1}{3}, \frac{1}{4}\right\rangle$ and the (additive) speedup term $\varphi=\frac{1}{16}$. (Note that $C_{1}$ is $\mathcal{C}$ 's slowest computer, and $C_{4}$ is its fastest.) Table 3 presents the work ratios obtained by speeding up each of $\mathcal{C}$ 's computers in turn by the additive term $\varphi$. Fig. 1 presents the same results graphically.

\begin{tabular}{|c|c|c|}
\hline & Profile & Work ratio \\
$i$ & $\mathrm{P}^{(i)}$ & $W\left(L ; \mathrm{P}^{(i)}\right) \div W(L ; \mathrm{P})$ \\
\hline \hline 1 & $\langle 15 / 16,1 / 2,1 / 3,1 / 4\rangle$ & 1.008 \\
\hline 2 & $\langle 1,7 / 16,1 / 3,1 / 4\rangle$ & 1.014 \\
\hline 3 & $\langle 1,1 / 2,13 / 48,1 / 4\rangle$ & 1.034 \\
\hline 4 & $\langle 1,1 / 2,1 / 3,3 / 16\rangle$ & 1.159 \\
\hline
\end{tabular}

Table 3: The work ratios as each of $\mathcal{C}$ 's 4 computers is sped up additively.

We see that one enhances $\mathcal{C}$ 's work production by $0.8 \%$ by speeding up the slowest computer, $C_{1}$, by $1.4 \%$ by speeding up the second slowest computer, $C_{2}$, by $3.4 \%$ by speeding up the second fastest computer, $C_{3}$, and by $15.9 \%$ by speeding up the fastest computer, $C_{4}$. Qualitatively similar results are observed with other clusters $\mathcal{C}$ and other speedup terms $\varphi$.

B. The multiplicative-speedup scenario. We compare two profiles: $\mathrm{P}^{[i]}$ is obtained by speeding up the slower computer (of the two we are focusing on), $C_{i}$; $\mathrm{P}^{[j]}$ is obtained by speeding up the faster one, $C_{j}$; both speedups are by the multiplicative factor $\psi<1$. We have

$$
\begin{aligned}
& \mathrm{P}^{[i]}=\left\langle\rho_{1}, \ldots, \rho_{i-1}, \quad \psi \rho_{i}, \quad \rho_{i+1}, \ldots, \rho_{j-1}, \quad \rho_{j}, \quad \rho_{j+1}, \ldots, \rho_{n}\right\rangle \\
& \mathrm{P}^{[j]}=\left\langle\rho_{1}, \ldots, \rho_{i-1}, \quad \rho_{i}, \quad \rho_{i+1}, \ldots, \rho_{j-1}, \quad \psi \rho_{j}, \quad \rho_{j+1}, \ldots, \rho_{n}\right\rangle
\end{aligned}
$$

The driving question of which computer to speed up has a more complicated answer in the multiplicative-speedup scenario than in the additive-speedup scenario.

Theorem 4. ${ }^{8}$ Let $\mathcal{C}$ contain computers $C_{i}$ and $C_{j}$, with respective computation rates $\rho_{i}$ and

\footnotetext{
${ }^{7}$ Proof appears in Section B.3.

${ }^{8}$ Proof appears in Section B.4.
} 


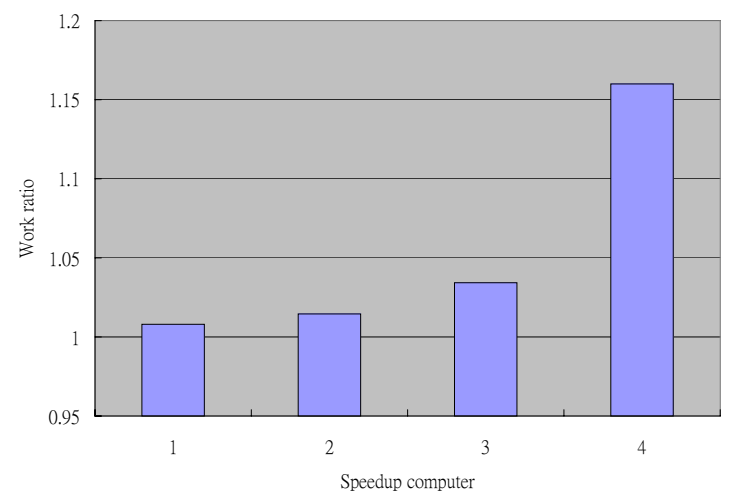

Figure 1: The work ratios as each of $\mathcal{C}$ 's 4 computers is sped up additively.

$\rho_{j}<\rho_{i}$. Under the multiplicative-speedup scenario with speedup factor $\psi$ :

- If $\psi \rho_{i} \rho_{j}>A \tau \delta / B^{2}$, then speeding up $C_{j}$ (the faster computer) allows one to complete more work than does speeding up $C_{i}$.

- If $\psi \rho_{i} \rho_{j}<A \tau \delta / B^{2}$, then speeding up $C_{i}$ (the slower computer) allows one to complete more work than does speeding up $C_{j}$.

Informal translation. It is more advantageous to speed up the faster computer, unless either both computers are already "very fast" or the speedup factor $\psi$ is "very small."

The values of "very fast" and "very small" depend on the relation between the problemspecific quantity $\psi \rho_{i} \rho_{j}$ and the environment-specific quantity $A \tau \delta / B^{2}$. For perspective, with our earlier values (see Table 1 ), we have $A \tau \delta / B^{2} \approx 1.1 \times 10^{-5}$; hence, we expect that speeding up the faster computer will usually be the better option.

Multiplicative speedup "in action." The experiment that illustrates multiplicative speedup "in action" is quite different from the one we used to illustrate additive speedup. We observe the two conditions of Theorem 4 "in action" via a sequence of snapshots of a cluster that experiences a sequence of multiplicative speedups. The snapshots depict a two-phase experiment that begins with a 4-computer homogeneous cluster $\mathcal{C}$ whose profile is $\mathrm{P}=\langle 1,1,1,1\rangle$ and that iteratively optimally speeds $\mathcal{C}$ up via the speedup factor $\psi=1 / 2$. The first phase illustrates the first condition in the proposition, as $\mathcal{C}$ 's profile "improves" (because of the speedups) from its initial value of $\mathrm{P}=\langle 1,1,1,1\rangle$ to the value $\mathrm{P}^{\prime}=\langle 5 / 80,5 / 80,5 / 80,5 / 80\rangle$. Once all of $\mathcal{C}$ 's computers achieve this speed, subsequent speedups follow the second condition in the proposition. Although we continue to speed up cluster $\mathcal{C}$ via the factor $\psi=1 / 2$, we observe the very different result predicted by the second condition. 
We have increased the value of the $\tau$ parameter for this experiment, from its earlier $1 \mu \mathrm{sec} /$ work unit to $200 \mu \mathrm{sec} /$ work unit. With the original value of $\tau$, the $\rho$-value of $\mathcal{C}$ 's fastest computer becomes too small to be seen when displayed with the $\rho$-values of the slower computers. Increasing the value of $\tau$ was an easy expedient for enhancing visibility while still illustrating the proposition.

Each bar-graph in Figs. 2 and 3 represents the then-current profile of $\mathcal{C}$ after one round of the experiment: when the four bars in each graph have respective heights $\rho_{1}, \rho_{2}, \rho_{3}$, and $\rho_{4}$, from left to right, this means that $\mathcal{C}$ 's prfile at that round is $\left\langle\rho_{1}, \rho_{2}, \rho_{3}, \rho_{4}\right\rangle$. The experiment proceeds as follows. Say that $\mathcal{C}$ has profile $\mathrm{P}_{i}$ after round $i$ of the experiment. At round $i+1$, we consider four potential successor profiles to profile $\mathrm{P}_{i}$, call them $\mathrm{P}_{i}^{[1]}, \mathrm{P}_{i}^{[2]}, \mathrm{P}_{i}^{[3]}$, and $\mathrm{P}_{i}^{[4]}$. Each profile $\mathrm{P}_{i}^{[j]}$ is obtained by speeding up only computer $C_{j}$ of $\mathcal{C}$, by the (multiplicative) factor $\psi=1 / 2$. We compare the work-productions of the four potential successor profiles, and we select the profile with the largest work-production to be profile $\mathrm{P}_{i}$ 's successor, $\mathrm{P}_{i+i}$. In case of ties - wherein speeding up computers $C_{j}$ and $C_{k}$ yield the same work-productionthen we choose to speed up the computer with the larger index. We discuss each phase in turn.

Phase 1: not all computers are "very fast." As we observe in Fig. 2, this phase of the ex-

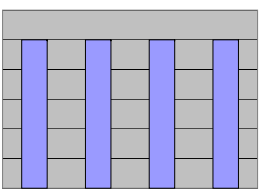

Round 1

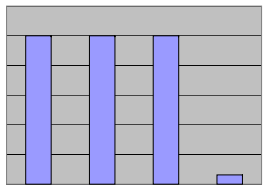

Round 5

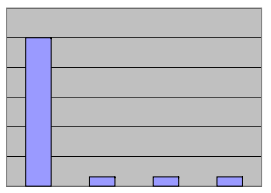

Round 13

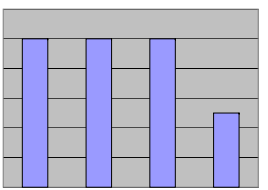

Round 2

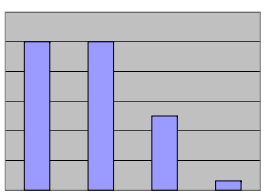

Round 6

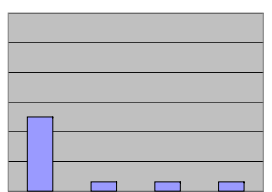

Round 14

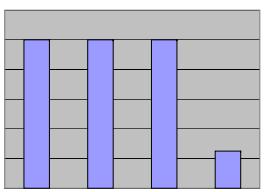

Round 3

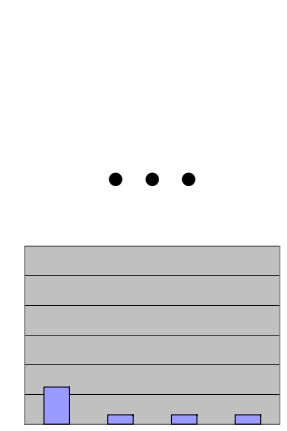

Round 15

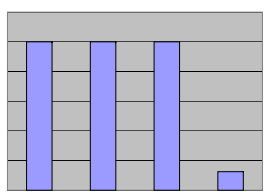

Round 4

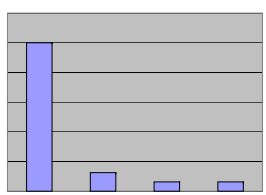

Round 12

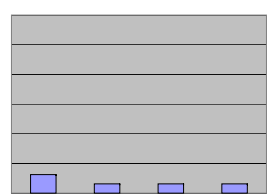

Round 16

Figure 2: Speeding up a cluster when not all computers are "very fast."

periment begins with an invocation of our tie-breaking mechanism because $\mathcal{C}$ is homogeneous before any speedups. We subsequently observe the repeated selection of the then-current fastest computer as the best one to speed up in rounds 2-16. Note that we choose to speed up computer $C_{4}$ in round 1 because of our tie-breaking mechanism, but we then select it in rounds $2-4$ because of the first condition in Theorem 4 . At round 5 , the second condition in 
Theorem 4 tells us not to speed up computer $C_{4}$ again. At that point, we again invoke the tie-breaking mechanism to select computer $C_{3}$, and the just-described cycle repeats, until $\mathcal{C}$ ends up in round 17 with the profile $\langle 0.0625,0.0625,0.0625,0.0625\rangle$. At this point, phase 2 of the experiment begins.

Phase 2: all computers are "very fast." At this point, the second condition in the proposition is triggered, and we observe the repeated selection of the slowest computer as the best one to speed up (with the tie-breaking mechanism used as necessary). Fig. 3 illustrates the pattern of speeding up a cluster under the second condition of Theorem 4.

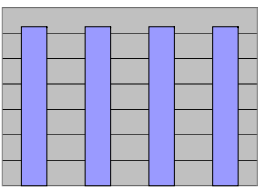

Round 17

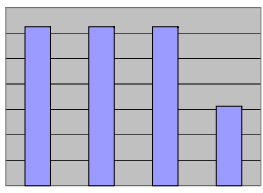

Round 18

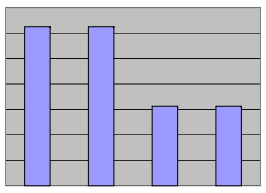

Round 19

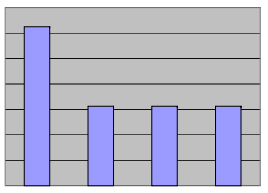

Round 20

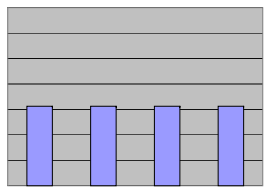

Round 21

Figure 3: Speeding up a cluster when all computers are "very fast."

\subsection{Predicting Power via Moments of Heterogeneity Profiles}

Proposition 2 tells us that if cluster $\mathcal{C}_{1}$ 's profile $\left\langle\rho_{1,1}, \ldots, \rho_{1, n}\right\rangle$ minorizes cluster $\mathcal{C}_{2}$ 's profile $\left\langle\rho_{2,1}, \ldots, \rho_{2, n}\right\rangle$, in the sense that $(a)$ for every index $i, \rho_{1, i} \leq \rho_{2, i},(b)$ for at least one index $i, \rho_{1, i}<\rho_{2, i}$, then $\mathcal{C}_{1}$ outperforms $\mathcal{C}_{2}$. It is easy to identify situations in which $\mathcal{C}_{1}$ outperforms $\mathcal{C}_{2}$ even though some of $\mathcal{C}_{1}$ 's computers are slower than any of $\mathcal{C}_{2}$ 's. For instance, a simple calculation shows that the cluster $\mathcal{C}_{1}$ with profile $\langle 0.99,0.02\rangle$ has a larger $X$-value than-hence, outperforms - the cluster $\mathcal{C}_{2}$ with profile $\langle 0.5,0.5\rangle$. This section is devoted to identifying situations in which the symmetric functions and (statistical) moments of two clusters' sets of $\rho$-values can be used to predict their relative performance. Regarding such moments: Note that the preceding 2-computer clusters show that having a better mean speed does not guarantee better performance.

A function $F\left(x_{1}, \ldots, x_{n}\right)$ is symmetric if its value is unchanged by every reordering of values for its variables. When $n=3$, for instance, we must have

$$
F(a, b, c)=F(a, c, b)=F(b, a, c)=F(b, c, a)=F(c, a, b)=F(c, b, a)
$$

for all values $a, b, c$ for the variables $x_{1}, x_{2}, x_{3}$. For integers $n>1$ and $k \in\{1, \ldots n\}$, we denote by $F_{k}^{(n)}$ the symmetric function that has $n$ variables grouped as products of $k$. It simplifies our analysis clerically if we allow the index $k$ to assume the value $k=0$ also, with the convention that, for all $n, F_{0}^{(n)} \equiv 1$. All of our values are computation rates $\rho_{i}$, so the first two families of $F_{k}^{(n)}$ functions (excluding the degenerate value $k=0$ ) are exhibited in Table 4. 


\begin{tabular}{|l|l|}
\hline$F_{1}^{(2)}\left(\rho_{1}, \rho_{2}\right)=\rho_{1}+\rho_{2}$ & $F_{1}^{(3)}\left(\rho_{1}, \rho_{2}, \rho_{3}\right)=\rho_{1}+\rho_{2}+\rho_{3}$ \\
$F_{2}^{(2)}\left(\rho_{1}, \rho_{2}\right)=\rho_{1} \rho_{2}$ & $F_{2}^{(3)}\left(\rho_{1}, \rho_{2}, \rho_{3}\right)=\rho_{1} \rho_{2}+\rho_{1} \rho_{3}+\rho_{2} \rho_{3}$ \\
& $F_{3}^{(3)}\left(\rho_{1}, \rho_{2}, \rho_{3}\right)=\rho_{1} \rho_{2} \rho_{3}$ \\
\hline
\end{tabular}

Table 4: The first two families of symmetric functions.

Note. There is a close relationship between some of the symmetric functions and standard statistical "moments." For any profile $\mathrm{P}=\left\langle\rho_{1}, \ldots, \rho_{n}\right\rangle$ :

- $F_{1}^{(n)}$ (resp., $F_{n}^{(n)}$ ) is the unnormalized arithmetic (resp., geometric) mean of the $\rho_{i}$.

- On the one hand, the variance of the $\rho_{i}$ is given by

$$
\begin{aligned}
\operatorname{VAR}(\mathrm{P}) & =\frac{1}{n}\left(\rho_{1}^{2}+\cdots+\rho_{n}^{2}\right)-\left(\frac{1}{n}\left(\rho_{1}+\cdots+\rho_{n}\right)\right)^{2} \\
\text { while } F_{2}^{(n)}(\mathrm{P}) & =\frac{1}{2}\left(\left(\rho_{1}+\cdots+\rho_{n}\right)^{2}-\left(\rho_{1}^{2}+\cdots+\rho_{n}^{2}\right)\right) .
\end{aligned}
$$

One can use the symmetric functions of clusters' profiles to compare the clusters' powers. We assume henceforth that $\tau \delta \leq A \leq B$. (Consider the semantics of our architectural parameters to see why this inequality is reasonable.)

Lemma 1. ${ }^{9}$ There exist positive constants, $\alpha_{0}, \alpha_{1}, \ldots, \alpha_{n-1}$ and $\beta_{0}, \beta_{1}, \ldots, \beta_{n}$, such that

$$
X(\mathrm{P})=\frac{\alpha_{0}+\alpha_{1} F_{1}^{(n)}(\mathrm{P})+\cdots+\alpha_{n-1} F_{n-1}^{(n)}(\mathrm{P})}{\beta_{0}+\beta_{1} F_{1}^{(n)}(\mathrm{P})+\cdots+\beta_{n-1} F_{n-1}^{(n)}(\mathrm{P})+\beta_{n} F_{n}^{(n)}(\mathrm{P})}
$$

Specifically: $\left\{\begin{array}{l}\text { for each } i \in\{0, \ldots, n-1\}, \quad \alpha_{i}=B^{i} \cdot \sum_{k=0}^{n-i-1} A^{k} \cdot(\tau \delta)^{n-k-i-1} \\ \text { for each } i \in\{0, \ldots, n\}, \quad \beta_{i}=B^{i} \cdot A^{n-i} .\end{array}\right.$

Expression (3.4) suggests a method for comparing profiles $\mathrm{P}_{1}$ and $\mathrm{P}_{2}$ by comparing their respective sets of symmetric functions.

Proposition 3. ${ }^{10}$ Let clusters $\mathcal{C}_{1}$ and $\mathcal{C}_{2}$ have, respectively, profiles $\mathrm{P}_{1}$ and $\mathrm{P}_{2}$. Cluster $\mathcal{C}_{1}$ outperforms cluster $\mathcal{C}_{2}$ whenever the following system of inequalities holds.

For all pairs of indices $i, j \in\{0, \ldots, n\}$, with $i<j$

$$
F_{i}^{(n)}\left(\mathrm{P}_{1}\right) \cdot F_{j}^{(n)}\left(\mathrm{P}_{2}\right) \geq F_{i}^{(n)}\left(\mathrm{P}_{2}\right) \cdot F_{j}^{(n)}\left(\mathrm{P}_{1}\right)
$$

and for at least one $i-j$ pair, the inequality is strict.

\footnotetext{
${ }^{9}$ Proof appears in Section B.5.

${ }^{10}$ Proof appears in Section B.6.
} 
Theorem 5. ${ }^{11}$ Say that cluster $\mathcal{C}_{1}$, with profile $\mathrm{P}_{1}$, and cluster $\mathcal{C}_{2}$, with profile $\mathrm{P}_{2}$, share the same mean speed. If cluster $\mathcal{C}_{1}$ outperforms cluster $\mathcal{C}_{2}$ because of the system of inequalities (3.5), then $\operatorname{VAR}\left(\mathrm{P}_{1}\right)>\operatorname{VAR}\left(\mathrm{P}_{2}\right)$. When $\mathcal{C}_{1}$ and $\mathcal{C}_{2}$ each has 2 computers, then the preceding sentence becomes a biconditional: $\mathcal{C}_{1}$ outperforms $\mathcal{C}_{2}$ if and only if $\operatorname{VAR}\left(\mathrm{P}_{1}\right)>\operatorname{VAR}\left(\mathrm{P}_{2}\right)$.

Corollary 1. Heterogeneity can actually lend power to a cluster. To wit, if one has two 2-computer clusters that share the same mean speed $-\mathcal{C}_{2}$, which is homogeneous, and $\mathcal{C}_{1}$, which is not-then $\mathcal{C}_{1}$ outperforms $\mathcal{C}_{2}$.

It would be exciting if the final sentence of Theorem 5 held for clusters of arbitrary sizes, not just $n=2$. This is an intuitively plausible hope because when $V A R\left(\mathrm{P}_{1}\right)>V A R\left(\mathrm{P}_{2}\right)$, one would expect $\mathrm{P}_{1}$ to contain some $\rho$-values that are smaller than any of $\mathrm{P}_{2}$ 's, and one would hope that these small values would pull $\mathcal{C}_{1}$ 's HECR down below $\mathcal{C}_{2}$ 's. (Because each $\rho_{i} \leq 1$, the small $\rho$-values should have greater impact on HECRs than do the large values.) But, alas, such is not the case. We performed the following simple experiment for $n$-computer clusters, for various integers $n$; each trial consisted of the following steps.

1. Randomly generate $n$-computer clusters $\mathcal{C}_{1}$ and $\mathcal{C}_{2}$, with respective profiles $\mathrm{P}_{1}$ and $\mathrm{P}_{2}$ and mean speeds $\bar{\rho}_{1}$ and $\bar{\rho}_{2}$.

(a) Alter the speeds of $\mathcal{C}_{2}$ 's computers by the factor $\bar{\rho}_{1} / \bar{\rho}_{2}$ (giving us cluster $\mathcal{C}_{2}^{\prime}$ with profile $\mathrm{P}_{2}^{\prime}$ ) so that $\mathcal{C}_{1}$ and $\mathcal{C}_{2}^{\prime}$ have the same mean speed (namely, $\bar{\rho}_{1}$ ).

(b) Reject the current pair if $\operatorname{VAR}\left(\mathrm{P}_{1}\right)=\operatorname{VAR}\left(\mathrm{P}_{2}^{\prime}\right)$ (which should be quite unlikely).

2. Compare the HECRs of $\mathcal{C}_{1}$ and $\mathcal{C}_{2}^{\prime}$. Label $\left(\mathcal{C}_{1}, \mathcal{C}_{2}^{\prime}\right)$ "good" if the cluster with larger variance has the smaller HECR (i.e, is more powerful); otherwise, label the pair "bad."

We found "bad" cluster-pairs for every size $n>2$. Moderating our disappointment is the fact that the clusters in the "bad" pairs had rather small differences in HECR. We therefore selected a variance threshold $\theta$, and we repeated a modified version of our experiment. Say, to be definite, with no loss of generality, that $\operatorname{VAR}\left(\mathrm{P}_{1}\right)>\operatorname{VAR}\left(\mathrm{P}_{2}^{\prime}\right)$. We replaced the condition "cluster with larger variance" - in this case, $\operatorname{VAR}\left(\mathrm{P}_{1}\right)>\operatorname{VAR}\left(\mathrm{P}_{2}^{\prime}\right)$-by the condition

"cluster whose variance is larger by at least $\theta$ " -in this case, $\operatorname{VAR}\left(\mathrm{P}_{1}\right) \geq \operatorname{VAR}\left(\mathrm{P}_{2}^{\prime}\right)+\theta$. Our goal was to find the smallest values of $\theta$ for which $\operatorname{HECR}\left(\mathcal{C}_{1}\right)<\operatorname{HECR}\left(\mathcal{C}_{2}^{\prime}\right) 100 \%$ of the time! We experimentally determined for pairs $\left(\mathcal{C}_{1}, \mathcal{C}_{2}^{\prime}\right)$ of 8-computer clusters:

Fact. Using the described experimental procedures, we observe $\operatorname{HECR}\left(\mathcal{C}_{1}\right)<\operatorname{HECR}\left(\mathcal{C}_{2}^{\prime}\right)$ $100 \%$ of the time when $\theta=0.15$, i.e., when $\operatorname{VAR}\left(\mathrm{P}_{1}\right)>\operatorname{VAR}\left(\mathrm{P}_{2}^{\prime}\right)+0.15$.

We thus have a version of Theorem 5's final sentence that, empirically, holds for 8-computer clusters. Ongoing experiments are extending this work to larger clusters, with the hope that $\theta_{n}$, the $n$-computers/cluster analogue of threshold $\theta\left(=\theta_{8}\right)$, grows slowly as a function of $n$.

\footnotetext{
${ }^{11}$ Proof appears in Section B.7.
} 


\section{Conclusions and Projections}

Heterogeneity is almost ubiquitous in modern computing platforms, yet sources such as [1] show that we have yet to unlock some very basic secrets about this phenomenon. One finds in [1] a simple computational problem (the CEP) all of whose optimal solutions for a given cluster $\mathcal{C}$ can be characterized (Theorem 1) and shown to be functions of $\mathcal{C}$ 's (heterogeneity) profile (Theorem 2). We build on these results to expose properties of $\mathcal{C}$ 's profile that determine the quality of solutions to the CEP for $\mathcal{C}$. Perhaps our most interesting resultscertainly our favorites - show the following: (1) If one can replace just one of $\mathcal{C}$ 's computers by a faster one, then: (a) If the new computer is additively faster than the old one, then the most advantageous computer to replace is $\mathcal{C}$ 's fastest one (Theorem 3). (b) The same is true for multiplicative speedups, unless either all of $\mathcal{C}$ 's computers are already "very fast" or the speedup factor is "very aggressive" (Theorem 4). (2) The symmetric functions of $\overline{\mathcal{C}}$ 's computers' speeds play a large role in determining $\mathcal{C}$ 's power (Lemma 1, Proposition 3), which suggests a similarly large role for the statistical moments of $\mathcal{C}$ 's computers' speeds (Theorem 5). (3) Heterogeneity can enhance the power of a cluster (Corollary 1). Ongoing research strives to better understand topics $(2,3)$, via experimentation and analysis.

\section{References}

[1] M. Adler, Y. Gong, A.L. Rosenberg (2008): On "exploiting" node-heterogeneous clusters optimally. Theory of Computing Systems 42, 465-487.

[2] T.E. Anderson, D.E. Culler, D.A. Patterson, and the NOW Team (1995): A case for NOW (networks of workstations). IEEE Micro 15, 54-64.

[3] M. Banikazemi, V. Moorthy, D.K. Panda (1998): Efficient collective communication on heterogeneous networks of workstations. ICPP'00, 460-467.

[4] C. Banino, O. Beaumont, L. Carter, J. Ferrante, A. Legrand, Y. Robert (2004): Scheduling strategies for master-slave tasking on heterogeneous processor grids. IEEE Trans. Parallel and Distr. Systs. 15, 319-330.

[5] O. Beaumont, L. Carter, J. Ferrante, A. Legrand, Y. Robert (2002): Bandwidth-centric allocation of independent tasks on heterogeneous platforms. IPDPS'02.

[6] O. Beaumont, A. Legrand, Y. Robert (2003): The master-slave paradigm with heterogeneous processors. IEEE Trans. Parallel and Distr. Systs. 14, 897-908.

[7] O. Beaumont, L. Marchal, Y. Robert (2005): Scheduling divisible loads with return messages on heterogeneous master-worker platforms. 12th Intl. High-Performance Computing Conf. LNCS 3769, Springer, Berlin, 498-507.

[8] P.B. Bhat, V.K. Prasanna, C.S. Raghavendra (1999): Efficient collective communication in distributed heterogeneous systems. ICDCS'g9. 
[9] R. Buyya, D. Abramson, J. Giddy (2001): A case for economy Grid architecture for service oriented Grid computing. $H C W^{\prime} 01$.

[10] R. Buyya, C.S. Yeo, S. Venugopal, J. Broberg, I. Brandic (2009): Cloud computing and emerging IT platforms: Vision, hype, and reality for delivering computing as the 5th utility. Future Generation Computer Systs., to appear.

[11] W. Cirne and K. Marzullo (1999): The Computational Co-Op: gathering clusters into a metacomputer. ICPP'99, 160-166.

[12] F. Cappello, P. Fraigniaud, B. Mans, A.L. Rosenberg (2005): An algorithmic model for heterogeneous clusters: rationale and experience. Intl. J. Foundations of Computer Science 16, $195-216$.

[13] P.-F. Dutot (2003): Master-slave tasking on heterogeneous processors. IPDPS'03.

[14] I. Foster and C. Kesselman [eds.] (2004): The Grid: Blueprint for a New Computing Infrastructure (2nd Ed.). Morgan-Kaufmann, San Francisco.

[15] P. Fraigniaud, B. Mans, A.L. Rosenberg (2005): Efficient trigger-broadcasting in heterogeneous clusters. J. Parallel and Distributed Computing 65 (2005) 628-642.

[16] E. Korpela, D. Werthimer, D. Anderson, J. Cobb, M. Lebofsky (2000): SETI@home: massively distributed computing for SETI. In Computing in Science and Engineering (P.F. Dubois, Ed.) IEEE Computer Soc. Press, Los Alamitos, CA.

[17] P. Liu and T.-H. Sheng (2000): Broadcast scheduling optimization for heterogeneous clusters systems. SPAA'00, 129-136.

[18] P. Liu and D.-W. Wang (2000): Reduction optimization in heterogeneous cluster environments. IPDPS'00.

[19] J. Mache, R. Broadhurst, J. Ely (2000): Ray tracing on cluster computers. PDPTA'00, 509515.

[20] G. Malewicz, A.L. Rosenberg, M. Yurkewych (2006): Toward a theory for scheduling DaGs in Internet-based computing. IEEE Trans. Comput. 55, 757-768.

[21] G.F. Pfister (1995): In Search of Clusters. Prentice-Hall.

[22] R. Prakash and D.K. Panda (1998): Designing communication strategies for heterogeneous parallel systems. Parallel Computing 24, 2035-2052.

[23] A.L. Rosenberg (1994): Needed: a theoretical basis for heterogeneous parallel computing. In Developing a Computer Science Agenda for High-Performance Computing (U. Vishkin, ed.), ACM Press, N.Y. (1994) 137-142.

[24] A.S. Tosun and A. Agarwal (2000): Efficient broadcast algorithms for heterogeneous networks of workstations. PDCS'00.

[25] S.W. White and D.C. Torney (1993): Use of a workstation cluster for the physical mapping of chromosomes. SIAM NEWS, March, 1993, 14-17. 


\section{A FIFO Protocols Allocate Work Proportionally}

How should one define work allocation that is proportional to computer speeds within the context of our model? Certainly, the parameters in our model will not permit the ideal notion of proportionality that is embodied in the equation $w_{i} / w_{i+1}=\rho_{i+1} / \rho_{i}$. The following result shows that FIFO-based work allocations do exhibit a strong level of proportionality in their work allocations. Inequality (A.1) is validated in Section A. Focus on a cluster $\mathcal{C}$ that has the heterogeneity profile $\mathrm{P}=\left\langle\rho_{1}, \ldots, \rho_{n}\right\rangle$, where $\rho_{1} \geq \cdots \geq \rho_{n}$.

Proposition 4. FIFO Protocols allocate work in proportion to computer speeds, in the following sense. If the FIFO protocol employs the startup indexing $s_{i}=i$ for all $i \in$ $\{1, \ldots, n-1\}$, then the work allocations satisfy

$$
\frac{\rho_{i+1}}{\rho_{i}}+A / B<\frac{w_{i}}{w_{i+1}}<(1+A / B+\tau / B) \cdot \frac{\rho_{i+1}}{\rho_{i}} .
$$

For perspective, using our sample parameter values, inequalities (A.1) become

$$
\begin{array}{ll}
\text { fine-grain tasks: } & \frac{\rho_{i+1}}{\rho_{i}}+0.0001<\frac{w_{i}}{w_{i+1}}<1.00012 \cdot \frac{\rho_{i+1}}{\rho_{i}} \\
\text { coarse-grain tasks: } & \frac{\rho_{i+1}}{\rho_{i}}+0.00001<\frac{w_{i}}{w_{i+1}}<1.000012 \cdot \frac{\rho_{i+1}}{\rho_{i}} .
\end{array}
$$

Proof. The proof of Theorem 2 in [1] actually gives more information than we have thus far indicated. Let cluster $\mathcal{C}$ that has the heterogeneity profile $\mathbf{P}=\left\langle\rho_{1}, \ldots, \rho_{n}\right\rangle$, where $\rho_{1} \geq$ $\cdots \geq \rho_{n}$. Let the FIFO protocol employ the startup indexing $s_{i}=i$ for all $i \in\{1, \ldots, n-1\}$. Then each work allocation $w_{i}$ (for computer $C_{i}$ ) is given exactly (i.e., not asymptotically) by:

$$
w_{i}=\left[\frac{1}{A+B \rho_{i}} \cdot \prod_{j=1}^{i-1} \frac{B \rho_{j}+\tau \delta}{A+B \rho_{j}}\right] \cdot(L-\tau \delta W(L ; \mathrm{P})-(n+1) \sigma) .
$$

(The parameter $\sigma$, which measures the cost of setting up an intercomputer communication, appears in the full model of [12], but not its asymptotic simplication.)

\begin{tabular}{|c|c|}
\hline \multicolumn{2}{|c|}{ Sample Values for Perspective } \\
\hline Quantity & Value \\
\hline$A / B$ (coarse tasks): & 0.0000101 \\
\hline$A / B$ (finer tasks): & 0.000101 \\
\hline $1+A / B+\tau / B$ (coarse tasks): & 1.0000111 \\
\hline $1+A / B+\tau / B$ (finer tasks): & 1.00011 \\
\hline
\end{tabular}

It follows that

$$
\frac{w_{i}}{w_{i+1}}=\frac{B \rho_{i+1}+A}{B \rho_{i}+A} \cdot \frac{B \rho_{i}+A}{B \rho_{i}+\tau \delta}=\frac{B \rho_{i+1}+A}{B \rho_{i}+\tau \delta}
$$

Elementary estimates then yield (A.1), because $A<B$ and both $\rho_{i}$ and $\rho_{i+1}$ are $\geq 1$. 


\section{B Proofs}

\section{B.1 Proof of Proposition 1}

By (2.2),

$$
X\left(\mathrm{P}^{(\rho)}\right)=\frac{1}{A-\tau \delta}\left(1-\left(\frac{B \rho+\tau \delta}{B \rho+A}\right)^{n}\right)
$$

By (B.2), then,

$$
\frac{B \rho+\tau \delta}{B \rho+A}=\left(1-(A-\tau \delta) X\left(\mathrm{P}^{(\rho)}\right)\right)^{1 / n}
$$

Therefore,

$$
B \rho+\tau \delta=(B \rho+A)\left(1-(A-\tau \delta) X\left(\mathrm{P}^{(\rho)}\right)\right)^{1 / n}
$$

so that

$$
B \rho\left(1-\left(1-(A-\tau \delta) X\left(\mathrm{P}^{(\rho)}\right)\right)^{1 / n}\right)=A\left(1-(A-\tau \delta) X\left(\mathrm{P}^{(\rho)}\right)\right)^{1 / n}-\tau \delta
$$

and

$$
\rho=\frac{1}{B} \cdot \frac{A\left(1-(A-\tau \delta) X\left(\mathrm{P}^{(\rho)}\right)\right)^{1 / n}-\tau \delta}{1-\left(1-(A-\tau \delta) X\left(\mathrm{P}^{(\rho)}\right)\right)^{1 / n}}
$$

Proposition 1 now follows via the following symbolic simplification. For all $D$, $\frac{A D-\tau \delta}{1-D}=\frac{A-\tau \delta}{1-D}-A$.

\section{B.2 Proof of Proposition 2}

Let profiles $\mathrm{P}$ and $\mathrm{P}^{\prime}$ be as in the statement of the proposition. We use a device from [1] to show that $X\left(\mathrm{P}^{\prime}\right)>X(\mathrm{P})$, so that $W\left(L ; \mathrm{P}^{\prime}\right)>(L ; \mathrm{P})$ for all $L$.

We begin by refining the expression $(2.2)$ for $X(\mathrm{P})$ to make explicit the startup order $\Sigma=$ $\left\langle s_{1}, \ldots, s_{n}\right\rangle$ used by $\mathcal{C}$. (By Theorem 1.2, this has no impact on $\mathcal{C}$ 's work production.) As we write $X(\mathrm{P} ; \Sigma)$ to announce the use of $\Sigma$, the only impact on $(2.2)$ is that the occurrence of " $\rho_{i}$ " in the expression becomes " $\rho_{s_{i}}$ " and the two occurrences of " $\rho_{j}$ " become " $\rho_{s_{j}}$." We next choose any startup order $\Sigma$ for $\mathcal{C}$, for which $s_{n}=i$; i.e., $\Sigma$ has the form $\Sigma=\left\langle s_{1}, \ldots, s_{n-1}, i\right\rangle$. We then form the appropriate versions of (2.2) that use startup order $\Sigma$. For the sake of perspicuity, we write these versions in the following way, which emphasize that $X(\mathrm{P} ; \Sigma)$ and $X\left(\mathrm{P}^{\prime} ; \Sigma\right)$ differ only in their first terms.

$$
\begin{aligned}
X(\mathrm{P} ; \Sigma) & =\frac{1}{A+B \rho_{s_{n}}} \prod_{j=1}^{n-1} \frac{B \rho_{s_{j}}+\tau \delta}{A+B \rho_{s_{j}}}+\sum_{i=1}^{n-1} \frac{1}{A+B \rho_{s_{i}}} \prod_{j=1}^{i-1} \frac{B \rho_{s_{j}}+\tau \delta}{A+B \rho_{s_{j}}} \\
X\left(\mathrm{P}^{\prime} ; \Sigma\right) & =\frac{1}{A+B \rho_{s_{n}}^{\prime}} \prod_{j=1}^{n-1} \frac{B \rho_{s_{j}}+\tau \delta}{A+B \rho_{s_{i}}}+\sum_{i=1}^{n-1} \frac{1}{A+B \rho_{s_{i}}} \prod_{j=1}^{i-1} \frac{B \rho_{s_{j}}+\tau \delta}{A+B \rho_{s_{j}}}
\end{aligned}
$$


Direct calculation now shows that

$$
X\left(\mathrm{P}^{\prime} ; \Sigma\right)-X(\mathrm{P} ; \Sigma)=\frac{B\left(\rho_{s_{n}}-\rho_{s_{n}}^{\prime}\right)}{\left(A+B \rho_{s_{n}}^{\prime}\right)\left(A+B \rho_{s_{n}}\right)} \cdot \prod_{j=1}^{n-1} \frac{B \rho_{j}+\tau \delta}{A+B \rho_{j}} .
$$

This difference is positive because $\rho_{s_{n}}=\rho_{i}>\rho_{i}^{\prime}=\rho_{s_{n}}^{\prime}$. We thus have $X\left(\mathrm{P}^{\prime} ; \Sigma\right)>X(\mathrm{P} ; \Sigma)$.

\section{B.3 Proof of Theorem 3}

As we compare $X\left(\mathrm{P}^{(i)}\right)$ and $X\left(\mathrm{P}^{(j)}\right)$, we lose no generality by using a startup ordering $\Sigma=\left\langle s_{1}, \ldots, s_{n-1}, s_{n}\right\rangle$ for $\mathcal{C}$ 's computers for which $s_{n}=i$ and $s_{n-1}=j$. We then obtain the following expressions via (3.1).

$$
\begin{aligned}
X\left(\mathrm{P}^{(i)}\right) & =\frac{A+B\left(\rho_{i}+\rho_{j}-\varphi\right)+\tau \delta}{A^{2}+A B\left(\rho_{i}+\rho_{j}-\varphi\right)+B^{2}\left(\rho_{i}-\varphi\right) \rho_{j}} \cdot Y(\mathrm{P})+Z(\mathrm{P}) \\
X\left(\mathrm{P}^{(j)}\right) & =\frac{A+B\left(\rho_{i}+\rho_{j}-\varphi\right)+\tau \delta}{A^{2}+A B\left(\rho_{i}+\rho_{j}-\varphi\right)+B^{2} \rho_{i}\left(\rho_{j}-\varphi\right)} \cdot Y(\mathrm{P})+Z(\mathrm{P})
\end{aligned}
$$

These expressions differ only in the terms $-B^{2} \varphi \rho_{j}$ and $-B^{2} \varphi \rho_{i}<-B^{2} \varphi \rho_{j}$ in the denominators of the lead fractions of $X\left(\mathrm{P}^{(i)}\right)$ and $X\left(\mathrm{P}^{(j)}\right)$, respectively. (The "lead fraction" in both expressions is the fraction that multiplies $Y(\mathrm{P})$.) Because $\rho_{i}>\rho_{j}$, it follows that $X\left(\mathrm{P}^{(j)}\right)>X\left(\mathrm{P}^{(i)}\right)$, whence the result.

\section{B.4 Proof of Theorem 4}

We have $\mathcal{C}$ employ the same startup order $\Sigma$ as we compare $X\left(\mathrm{P}^{[i]}\right)$ and $X\left(\mathrm{P}^{[j]}\right)$ as we did when we compared $X\left(\mathrm{P}^{(i)}\right)$ and $X\left(\mathrm{P}^{(j)}\right)$ (in Section B.3); hence, $s_{n}=i$ and $s_{n-1}=j$. Specializing (3.1) therefore yields

$$
\begin{aligned}
X\left(\mathrm{P}^{[i]}\right) & =\frac{A+B\left(\psi \rho_{i}+\rho_{j}\right)+\tau \delta}{A^{2}+A B\left(\psi \rho_{i}+\rho_{j}\right)+B^{2} \psi \rho_{i} \rho_{j}} \cdot Y(\mathrm{P})+Z(\mathrm{P}) \\
X\left(\mathrm{P}^{[j]}\right) & =\frac{A+B\left(\rho_{i}+\psi \rho_{j}\right)+\tau \delta}{A^{2}+A B\left(\rho_{i}+\psi \rho_{j}\right)+B^{2} \psi \rho_{i} \rho_{j}} \cdot Y(\mathrm{P})+Z(\mathrm{P})
\end{aligned}
$$

Clearly, then, we have $X\left(\mathrm{P}^{[i]}\right)>X\left(\mathrm{P}^{[j]}\right)$ (resp., $\left.X\left(\mathrm{P}^{[j]}\right)>X\left(\mathrm{P}^{[i]}\right)\right)$ if, and only if,

$$
\Upsilon^{[i]} \stackrel{\text { def }}{=} \frac{A+B\left(\psi \rho_{i}+\rho_{j}\right)+\tau \delta}{A^{2}+A B\left(\psi \rho_{i}+\rho_{j}\right)+B^{2} \psi \rho_{i} \rho_{j}}>\Upsilon^{[j]} \stackrel{\text { def }}{=} \frac{A+B\left(\rho_{i}+\psi \rho_{j}\right)+\tau \delta}{A^{2}+A B\left(\rho_{i}+\psi \rho_{j}\right)+B^{2} \psi \rho_{i} \rho_{j}}
$$


(resp., $\Upsilon^{[j]}>\Upsilon^{[i]}$ ). By "cross-multiplying" to eliminate the fractions, we note finally that $\Upsilon^{[i]}>\Upsilon^{[j]}$ (resp., $\Upsilon^{[j]}>\Upsilon^{[i]}$ ) if, and only if, $\Xi^{[i]}>\Xi^{[j]}$ (resp., $\Xi^{[j]}>\Xi^{[i]}$ ) where

$$
\begin{aligned}
\Xi^{[i]}= & A^{3}+A^{2} B\left(\psi \rho_{i}+\rho_{j}\right)+A^{2} \tau \delta \\
& +A^{2} B\left(\rho_{i}+\psi \rho_{j}\right)+A B^{2}\left(\psi \rho_{i}+\rho_{j}\right)\left(\rho_{i}+\psi \rho_{j}\right)+A B\left(\rho_{i}+\psi \rho_{j}\right) \tau \delta \\
& +A B^{2} \psi \rho_{i} \rho_{j}+B^{3} \psi \rho_{i} \rho_{j}\left(\psi \rho_{i}+\rho_{j}\right)+B^{2} \psi \rho_{i} \rho_{j} \tau \delta \\
\Xi^{[j]}= & A^{3}+A^{2} B\left(\rho_{i}+\psi \rho_{j}\right)+A^{2} \tau \delta \\
& +A^{2} B\left(\psi \rho_{i}+\rho_{j}\right)+A B^{2}\left(\psi \rho_{i}+\rho_{j}\right)\left(\rho_{i}+\psi \rho_{j}\right)+A B\left(\psi \rho_{i}+\rho_{j}\right) \tau \delta \\
& +A B^{2} \psi \rho_{i} \rho_{j}+B^{3} \psi \rho_{i} \rho_{j}\left(\rho_{i}+\psi \rho_{j}\right)+B^{2} \psi \rho_{i} \rho_{j} \tau \delta
\end{aligned}
$$

Because $\psi<1$ and $\rho_{i}>\rho_{j}$, the result follows by considering when the difference

$$
\Xi^{[j]}-\Xi^{[i]}=\left[\left(B^{2} \psi \rho_{i} \rho_{j}-A \tau \delta\right) B\right]\left[(1-\psi)\left(\rho_{i}-\rho_{j}\right)\right]
$$

is positive and when it is negative.

\section{B.5 Proof of Lemma 1}

Focus on a fixed, but arbitrary profile $\mathrm{P}=\left\langle\rho_{1}, \ldots, \rho_{n}\right\rangle$, and expand (2.2) to express $X(\mathrm{P})$ as a single fraction, $X(\mathrm{P})=X_{\text {num }} / X_{\text {denom }}$.

Analyzing $X_{\text {denom }}$. Consider first the denominator, $X_{\text {denom }}$, of the fraction, which is simpler to analyze than the numerator. Easily, $X_{\text {denom }}$ is the $n$-factor product $X_{\text {denom }}=$ $\prod_{i=1}^{n}\left(B \rho_{i}+A\right)$. Using reasoning analogous to the proof of the Binomial Theorem, it is clear that, for each $i \in\{0, \ldots, n\}$, the coefficient, $\beta_{i}$, of $F_{i}(\mathrm{P})$ in $X_{\text {denom }}$ is $\beta_{i}=B^{i} \cdot A^{n-i}$.

Analyzing $X_{\text {num. }}$. We begin to analyze the numerator, $X_{\text {num }}$, of the fraction by expressing it as an $n$-term sum of products, where each product can be factored into an " $I$ - $J$ product," as follows.

$$
X_{\text {num }}=\sum_{j=1}^{n} I_{j} \cdot J_{j} \quad \text { where } \quad I_{j}=\prod_{k=j+1}^{n}\left(B \rho_{k}+A\right) \quad \text { and } \quad J_{j}=\prod_{k=1}^{j-1}\left(B \rho_{k}+\tau \delta\right) .
$$

Note that, for each $j \in\{0, \ldots, n\}$, the $j$ th $I-J$ product, $I_{j} \cdot J_{j}$, is the unique one that does not "mention" $\rho_{j}$.

Focus now on an arbitrary $i \in\{0, \ldots, n\}$ and an arbitrary $i$-monomial $\mu=\rho_{k_{1}} \cdots \rho_{k_{i}}$. Consider the coefficient of $\mu$ in $F_{i}(\mathrm{P})$. As just noted, $\mu$ appears as a subproduct of every $I-J$ product $I_{\ell} \cdot J_{\ell}$ where $\ell \in\{0, \ldots, n\} \backslash\left\{k_{1}, \ldots, k_{i}\right\}$; focus on an arbitrary such index $\ell$. Say that $\mu$ is "split" between $I_{\ell}$ and $J_{\ell}$, in the sense that $0 \leq h \leq i$ of the $\rho$-values that appear in $\mu$ are "mentioned" in $I_{\ell}$, and the other $i-h \rho$-values are "mentioned" in $J_{\ell}$. (The extreme cases, $h=0$ and $h=i$, correspond, respectively, to $\mu$ 's being a subproduct of $J_{\ell}$ or 
$I_{\ell}$.) Reasoning analogous to that used in analyzing $X_{\text {denom }}$ shows that $\mu$ 's coefficient in the product $I_{\ell} \cdot J_{\ell}$ is

$$
B^{i} \cdot\left(A^{n-h-\ell} \cdot(\tau \delta)^{\ell-(i-h)-1}\right) .
$$

Next, note that, given $\mu$, the coefficient (B.3) identifies index $\ell$ uniquely. Note also that, for each of the $i+1$ possible values for $h$, there is an $I-J$ product containing $\mu$ as a subproduct, within which $\mu$ provides $h \rho$-values to the $I$-portion of the product and $i-h \rho$-values to the $J$-portion. The just-exposed correspondences between $I-J$ products and monomials and conversely allow us to conclude that the coefficient of $F_{i}(\mathrm{P})$ in $X_{\text {num }}$ is a sum over $I-J$ products, whose summands represent allocations of monomials the the $I$ and $J$ portions of the products. In detail: for each $i, \alpha_{i}=B^{i} \cdot \sum_{k=0}^{n-i-1} A^{k} \cdot(\tau \delta)^{n-k-i-1}$.

\section{B.6 Proof of Proposition 3}

After "cross-multiplying" the fractions in expression (3.4), we see that $X\left(\mathrm{P}_{1}\right)>X\left(\mathrm{P}_{2}\right)$ if, and only if, the following " $\alpha-\beta$ difference" is positive:

$$
\begin{aligned}
& \left(\alpha_{0} F_{0}^{(n)}\left(\mathrm{P}_{1}\right)+\cdots+\alpha_{n-1} F_{n-1}^{(n)}\left(\mathrm{P}_{1}\right)\right) \cdot\left(\beta_{0} F_{0}^{(n)}\left(\mathrm{P}_{2}\right)+\cdots+\beta_{n} F_{n}^{(n)}\left(\mathrm{P}_{2}\right)\right) \\
& \quad-\left(\alpha_{0} F_{0}^{(n)}\left(\mathrm{P}_{2}\right)+\cdots+\alpha_{n-1} F_{n-1}^{(n)}\left(\mathrm{P}_{2}\right)\right) \cdot\left(\beta_{0} F_{0}^{(n)}\left(\mathrm{P}_{1}\right)+\cdots+\beta_{n} F_{n}^{(n)}\left(\mathrm{P}_{1}\right)\right)
\end{aligned}
$$

Consider now arbitrary indices $i, j \in\{0, \ldots, n\}$, with $i<j$, and focus on the portion of the " $\alpha$ - $\beta$ difference" that involves exactly the four quantities $F_{i}^{(n)}\left(\mathrm{P}_{1}\right), F_{i}^{(n)}\left(\mathrm{P}_{1}\right), F_{j}^{(n)}\left(\mathrm{P}_{2}\right)$, and $F_{j}^{(n)}\left(\mathrm{P}_{2}\right)$. One sees easily that this portion of the difference is precisely the product

$$
\left(\alpha_{i} \beta_{j}-\alpha_{j} \beta_{i}\right) \cdot\left(F_{i}^{(n)}\left(\mathrm{P}_{1}\right) \cdot F_{j}^{(n)}\left(\mathrm{P}_{2}\right)-F_{i}^{(n)}\left(\mathrm{P}_{2}\right) \cdot F_{j}^{(n)}\left(\mathrm{P}_{1}\right)\right)
$$

The following result will allow us to complete the proof.

$$
\text { Claim. For all indices } i \text { and } j>i \quad \alpha_{i} \beta_{j}>\alpha_{j} \beta_{i}
$$

We verify claim (B.5) by direct calculation. From Lemma 1, we know that

$$
\left[\alpha_{i}=B^{i} \cdot \sum_{k=0}^{n-1-i} A^{n-1-k-i} \cdot(\tau \delta)^{k}\right] \text { and }\left[\beta_{i}=B^{i} \cdot A^{n-i}\right]
$$


It follows that

$$
\begin{aligned}
\alpha_{i} \beta_{j}-\alpha_{j} \beta_{i}= & {\left[B^{i} \cdot \sum_{k=0}^{n-1-i} A^{n-1-k-i} \cdot(\tau \delta)^{k}\right] \cdot\left[B^{j} \cdot A^{n-j}\right] } \\
& \quad-\left[B^{j} \cdot \sum_{k=0}^{n-1-j} A^{n-1-k-j} \cdot(\tau \delta)^{k}\right] \cdot\left[B^{i} \cdot A^{n-i}\right] \\
= & B^{i+j} \cdot\left(\sum_{k=0}^{n-1-i} A^{2 n-1-k-i-j} \cdot(\tau \delta)^{k}-\sum_{k=0}^{n-1-j} A^{2 n-1-k-j-i} \cdot(\tau \delta)^{k}\right) \\
= & B^{i+j} \cdot \sum_{k=n-j}^{n-1-i} A^{2 n-1-k-i-j} \cdot(\tau \delta)^{k} \\
> & 0
\end{aligned}
$$

The last inequality holds because every term in the last summation is positive. This verifies claim (B.5).

To complete the argument, note that whenever (B.5) holds for a pair of indices $i$ and $j$, the product (B.4) is positive whenever (in fact, precisely when) the difference

$$
F_{i}^{(n)}\left(\mathrm{P}_{1}\right) \cdot F_{j}^{(n)}\left(\mathrm{P}_{2}\right)-F_{i}^{(n)}\left(\mathrm{P}_{2}\right) \cdot F_{j}^{(n)}\left(\mathrm{P}_{1}\right)
$$

is positive. Because (B.5) in fact holds for all $i$ and $j>i$, we see that the " $\alpha$ - $\beta$ difference" is positive whenever (3.5) holds. This means, however, that $X\left(\mathrm{P}_{1}\right)>X\left(\mathrm{P}_{2}\right)$ whenever (3.5) holds, whence the proposition.

\section{B.7 Proof of Theorem 5}

Let $\mathrm{P}_{1}=\left\langle\rho_{11}, \ldots, \rho_{1 n}\right\rangle$ and $\mathrm{P}_{2}=\left\langle\rho_{21}, \ldots, \rho_{2 n}\right\rangle$. By $(3.2)$, if $F_{1}^{(n)}\left(\mathrm{P}_{1}\right)=F_{1}^{(n)}\left(\mathrm{P}_{2}\right)$, then:

$$
\left[V A R\left(\mathrm{P}_{1}\right)>\operatorname{VAR}\left(\mathrm{P}_{2}\right)\right] \text { if, and only if, }\left[\rho_{11}^{2}+\cdots+\rho_{1 n}^{2}>\rho_{21}^{2}+\cdots+\rho_{2 n}^{2}\right] \text {. }
$$

But we know that $\left(\rho_{11}+\cdots+\rho_{1 n}\right)^{2}=\left(\rho_{21}+\cdots+\rho_{2 n}\right)^{2}$ (because of the equal mean speeds); hence we have, by $(3.3),\left[F_{2}^{(n)}\left(\mathrm{P}_{1}\right)<F_{2}^{(n)}\left(\mathrm{P}_{2}\right)\right]$.

When $n=2$, there are only two symmetric functions, $F_{1}^{(2)}$ and $F_{2}^{(2)}$, so the relations between the clusters' mean speeds and variances determine the relations between their profiles' symmetric functions. 